TRANSACTIONS OF THE

AMERICAN MATHEMATICAL SOCIETY

Volume 350, Number 7, July 1998, Pages 2879-2902

S $0002-9947(98) 02096-0$

\title{
BOUNDS FOR MULTIPLICITIES
}

\author{
JÜRGEN HERZOG AND HEMA SRINIVASAN
}

\begin{abstract}
Let $R=K\left[x_{1}, x_{2}, \ldots, x_{n}\right]$ and $S=R / I$ be a homogeneous $K$ algebra. We establish bounds for the multiplicity of certain homogeneous $K$-algebras $S$ in terms of the shifts in a free resolution of $S$ over $R$. Huneke and we conjectured these bounds as they generalize the formula of Huneke and Miller for the algebras with pure resolution, the simplest case. We prove these conjectured bounds for various algebras including algebras with quasipure resolutions. Our proof for this case gives a new and simple proof of the Huneke-Miller formula. We also settle these conjectures for stable and square free strongly stable monomial ideals $I$. As a consequence, we get a bound for the regularity of $S$. Further, when $S$ is not Cohen-Macaulay, we show that the conjectured lower bound fails and prove the upper bound for almost Cohen-Macaulay algebras as well as algebras with a $p$-linear resolution.
\end{abstract}

\section{INTRODUCTION}

This paper is inspired by a beautiful formula (in [13] and [4]) of Huneke and Miller for the multiplicity of rings with pure resolutions.

Throughout this paper, we fix a field $K$, and let $S$ be a homogeneous $K$-algebra. In other words, $S$ is a finitely generated graded $K$-algebra, generated over $K$ by elements of degree 1 , and hence is isomorphic to $R / I$ where $R=K\left[x_{1}, \ldots, x_{n}\right]$ is a polynomial ring and $I$ is a graded ideal contained in $\left(x_{1}, \ldots, x_{n}\right)$. Consider the graded minimal free resolution of $S$ :

$$
0 \longrightarrow \bigoplus_{j=1}^{b_{s}} R\left(-d_{s j}\right) \longrightarrow \cdots \longrightarrow \bigoplus_{j=1}^{b_{1}} R\left(-d_{1 j}\right) \longrightarrow R \longrightarrow 0 .
$$

The ring $S$ is said to have a pure resolution if for all $j$, the $d_{j i}$ do not depend on $i$ (but only on $j$ ). Hence if the resolution is pure it has the following shape:

$$
0 \longrightarrow R\left(-d_{s}\right)^{b_{s}} \longrightarrow \cdots \longrightarrow R\left(-d_{1}\right)^{b_{1}} \longrightarrow R \longrightarrow 0 .
$$

When $S$ is a Cohen-Macaulay ring with a pure resolution, Huneke and Miller's formula states that the multiplicity $e$ of $S$ is

$$
e(S)=\left(\prod_{i=1}^{s} d_{i}\right) / s !
$$

In general we define $M_{i}=\max \left\{d_{i j}: j=1, \ldots, b_{i}\right\}$ and $m_{i}=\min \left\{d_{i j}: j=\right.$ $\left.1, \ldots, b_{i}\right\}$ for $i=1, \ldots, s$.

Received by the editors July 4, 1996.

1991 Mathematics Subject Classification. Primary 13H15, 13D25, 13Xxx.

The second author was supported in part by grants from National Science Foundation and U.M. Research Board. 
Huneke and the second author conjectured the following:

Conjecture 1. For each homogeneous Cohen-Macaulay K-algebra $S$

$$
\left(\prod_{i=1}^{s} m_{i}\right) / s ! \leq e(S) \leq\left(\prod_{i=1}^{s} M_{i}\right) / s !
$$

Notice that the conjecture is true for complete intersections. Indeed, let $y_{1}, \ldots, y_{s}$ be a homogeneous regular sequence generating $I$. Let $a_{i}$ be the degree of $y_{i}$ for $i=1, \ldots, s$. We may assume that $a_{1} \leq a_{2} \leq \cdots \leq a_{s}$. Since the Koszul complex associated with this sequence provides a resolution of $R / I$ we have

$$
m_{i}=a_{1}+\ldots+a_{i} \quad \text { and } \quad M_{i}=a_{s-i+1}+\ldots+a_{s}
$$

for $i=1, \ldots, s$.

On the other hand we have $e(R / I)=\prod_{i=1}^{s} a_{i}$. Hence it follows that

$$
\begin{aligned}
\left(\prod_{i=1}^{s} m_{i}\right) / s ! & \leq\left(\prod_{i=1}^{s} i a_{i}\right) / s !=e(R / I) \\
& \leq\left(\prod_{i=1}^{s} i a_{s-i+1}\right) / s ! \leq\left(\prod_{i=1}^{s} M_{i}\right) / s !
\end{aligned}
$$

We call a resolution quasi-pure if the $m_{i} \geq M_{i-1}$ for all $i$, so that there is at most one shift common to any two consecutive modules in the resolution. We prove the conjecture for Cohen-Macaulay rings with quasi-pure resolutions. As a consequence, we get a simpler proof of the Huneke-Miller formula. Our proof of the conjecture in the quasi-pure case uses solely the equations satisfied by the shifts $d_{i j}$ in the resolution (Lemma 1.1 and Theorem 1.2). These relations by themselves will not imply the conjecture in general. This is evident even in codimension 2. Using a different analysis, we prove the conjecture for $R / I$ when $I$ is a stable ideal in the sense of Eliahou and Kervaire [9]; or a squarefree strongly stable ideal in the sense of Aramova, Herzog and Hibi [2].

In fact, we show that the conjecture is true in each of the following cases.

(i) $S$ has a quasi-pure resolution;

(ii) $I$ is a perfect ideal of codimension 2, or a codimension 3 Gorenstein ideal generated by 5 elements. (The upper conjectured inequality is proved for all codimension 3 Gorenstein ideals);

(iii) $I$ is a Gorenstein monomial ideal of codimension 3 with at least one generator of smallest possible degree (relative to the number of generators);

(iv) $I$ is a stable ideal;

(v) $I$ is a squarefree strongly stable ideal.

The lower bound in Conjecture 1 fails in general if $S$ is not Cohen-Macaulay. Even the smaller product $\prod_{i=1}^{h} m_{i}$ with $h=\operatorname{codim} S$ is in general not a lower bound for the multiplicity. Indeed, we show in Theorem 1.5 that in the best possible non-Cohen-Macaulay case, namely when $S$ has a pure resolution and $\operatorname{codim} S=$ codepth $S-1=s-1$, one has

$$
e(S)<\left(\prod_{i=1}^{s-1} m_{i}\right) /(s-1) !
$$

On the other hand, in the cases (iv) and (v) the upper bound is valid without the hypothesis that $S$ be Cohen-Macaulay. Thus we are tempted to conjecture that 
the upper bound is valid for any homogeneous $K$-algebra $S$. Actually, we expect the following stronger inequality

Conjecture 2. Let $S$ be a homogeneous $K$-algebra of codimension $h$. Then

$$
e(S) \leq\left(\prod_{i=1}^{h} M_{i}\right) / h !
$$

When $S$ is Cohen-Macaulay this is the same upper bound as in Conjecture 1, and is stronger otherwise. We show that Conjecture 2 is valid if the defining ideal of $S$ is stable, or squarefree strongly stable, or if $S$ has a $p$-linear resolution

$$
0 \longrightarrow R(-p-s+1)^{b_{s}} \longrightarrow \cdots \longrightarrow R(-p-1)^{b_{2}} \longrightarrow R(-p)^{b_{1}} \longrightarrow R \longrightarrow 0,
$$

but is not necessarily Cohen-Macaulay.

Combining (iv) with a theorem of Bayer and Stillman [6] we obtain the following quite general inequality: Let $I \subset R$ be any graded ideal of codim $s$, then

$$
e(R / I) \leq\left(\begin{array}{c}
\operatorname{reg}(I)+s-1 \\
s
\end{array}\right)
$$

where $\operatorname{reg}(I)$ denotes the Castelnuovo-Mumford regularity. This formula has been recently improved by N. Terai [15].

\section{QUASI-PURE RESOLUTIONS}

The purpose of this section is to prove the conjecture for quasi-pure resolutions. This provides a simple proof of the Huneke-Miller formula. We also prove the upper conjectured inequality for multiplicities for rings with $p$-linear resolution.

Throughout this paper $R=K\left[x_{1}, \ldots, x_{n}\right]$ will denote the polynomial ring in $n$ variables defined over a field $K, I \subset R$ will be a graded ideal, and $S=R / I$ the residue class ring modulo $I$. Furthermore, we let

$$
0 \longrightarrow \bigoplus_{j=1}^{b_{s}} R\left(-d_{s j}\right) \longrightarrow \cdots \longrightarrow \bigoplus_{j=1}^{b_{1}} R\left(-d_{1 j}\right) \longrightarrow R \longrightarrow 0
$$

be the resolution of $S$.

Given $S$ with the resolution as above, we define $M_{i}=\max \left\{d_{i j}: j=1, \ldots, b_{i}\right\}$ and $m_{i}=\min \left\{d_{i j}: j=1, \ldots, b_{i}\right\}$ for $i=1, \ldots, s$ to be the maximal and minimal shifts respectively in the resolution.

The next result has probably first been observed by Peskine and Szpiro [14].

Lemma 1.1. With the above notation, we have

$$
\sum_{i=1}^{s}(-1)^{i} \sum_{j=1}^{b_{i}} d_{i j}^{k}= \begin{cases}0 & \text { for } 1 \leq k<r, \\ (-1)^{r} r ! e(S) & \text { for } k=r,\end{cases}
$$

where $r$ is the codimension of $S$.

Proof. From the free $R$-resolution of $S$ we deduce that the Hilbert series of $S$ has the form

$$
H_{S}(t)=\frac{\sum_{i=0}^{s}(-1)^{i} \sum_{j=1}^{b_{i}} t^{d_{i j}}}{(1-t)^{n}} .
$$

On the other hand the Hilbert series is known to be a rational function of the form

$$
\frac{Q(t)}{(1-t)^{d}}
$$

where $d=\operatorname{dim} S$ and where $Q(1)=e$. 
A comparison of these two formulas yields the equation

$$
\sum_{i=0}^{s}(-1)^{i} \sum_{j=1}^{b_{i}} t^{d_{i j}}=Q(t)(1-t)^{r} .
$$

Denoting the polynomial on the left hand side of this equation by $P(t)$, we see that for the higher formal derivatives $P^{(k)}(t)$ of $P(t)$ we have

$$
P^{(k)}(1)= \begin{cases}0 & \text { for } 1 \leq j<r \\ (-1)^{r} r ! e(S) & \text { for } j=r\end{cases}
$$

From this one deduces the assertion.

Theorem 1.2. Suppose that $S$ is a Cohen-Macaulay with a quasi-pure resolution. Then Conjecture 1 holds. That is,

$$
\left(\prod_{i=1}^{s} m_{i}\right) / s ! \leq e(S) \leq\left(\prod_{i=1}^{s} M_{i}\right) / s ! .
$$

Proof. Consider the square matrix

$$
M=\left(\begin{array}{ccccc}
\sum_{j=1}^{b_{1}} d_{1 j} & \sum_{j=1}^{b_{2}} d_{2 j} & \cdots & \sum_{j=1}^{b_{s-1}} d_{s-1 j} & \sum_{j=1}^{b_{s}} d_{s j} \\
\sum_{j=1}^{b_{1}} d_{1 j}^{2} & \sum_{j=1}^{b_{2}} d_{2 j}^{2} & \cdots & \sum_{j=1}^{b_{s-1}} d_{s-1 j}^{2} & \sum_{j=1}^{b_{s}} d_{s j}^{2} \\
\vdots & \vdots & \vdots & \vdots & \vdots \\
\sum_{j=1}^{b_{1}} d_{1 j}^{s} & \sum_{j=1}^{b_{2}} d_{2 j}^{s} & \cdots & \sum_{j=1}^{b_{s-1}} d_{s-1 j}^{s} & \sum_{j=1}^{b_{s}} d_{s j}^{s}
\end{array}\right)
$$

of size $s$.

We will compute the determinant $|M|$ of $M$ in two different ways. First we replace the last column of $M$ by the alternating sum of all columns of $M$. The resulting matrix will be denoted by $M^{\prime}$. It is clear that $|M|=(-1)^{s}\left|M^{\prime}\right|$. Moreover, due to 1.1 , the last column of $M^{\prime}$ is the transpose of the vector $\left(0, \ldots, 0,(-1)^{s} s ! e(S)\right)$. Thus if we expand $M^{\prime}$ with respect to the last column we get

$$
|M|=(-1)^{s}\left|M^{\prime}\right|=s ! e(S)|N|
$$

where $N$ is the matrix

$$
\left(\begin{array}{cccc}
\sum_{j=1}^{b_{1}} d_{1 j} & \sum_{j=1}^{b_{2}} d_{2 j} & \cdots & \sum_{j=1}^{b_{s-1}} d_{s-1 j} \\
\sum_{j=1}^{b_{1}} d_{1 j}^{2} & \sum_{j=1}^{b_{2}} d_{2 j}^{2} & \cdots & \sum_{j=1}^{b_{s-1}} d_{s-1 j}^{2} \\
\vdots & \vdots & \vdots & \vdots \\
\sum_{j=1}^{b_{1}} d_{1 j}^{s-1} & \sum_{j=1}^{b_{2}} d_{2 j}^{s-1} & \cdots & \sum_{j=1}^{b_{s-1}} d_{s-1 j}^{s-1}
\end{array}\right)
$$

of size $s-1$.

On the other hand,

$$
|M|=\sum_{\substack{1 \leq j_{i} \leq b_{i} \\ 1 \leq i \leq s}} \prod_{i=1}^{s} d_{i j_{i}} V\left(d_{1 j_{1}}, \ldots, d_{s j_{s}}\right),
$$


where

$$
V\left(d_{1 j_{1}}, \ldots, d_{s j_{s}}\right)=\left|\begin{array}{ccccc}
1 & 1 & \cdots & 1 & 1 \\
d_{1 j_{1}} & d_{2 j_{2}} & \cdots & d_{s-1 j_{s-1}} & d_{s j_{s}} \\
\vdots & \vdots & \vdots & \vdots & \vdots \\
d_{1 j_{1}}^{s-1} & d_{2 j_{2}}^{s-1} & \cdots & d_{s-1 j_{s-1}}^{s-1} & d_{s j_{s}}^{s-1}
\end{array}\right|
$$

is the Vandermonde determinant.

We note that all the Vandermonde determinants in the sum (2) are nonnegative because the resolution is quasi-pure, so that $d_{i j} \geq d_{i-1 k}$ for all $i, j$ and $k$. Replacing the products of the $d_{i j_{i}}$ by their minimum and maximum, we obtain

$$
\begin{aligned}
& \prod_{i=1}^{s} M_{i} \sum_{\substack{1 \leq j_{i} \leq b_{i} \\
1 \leq i \leq s}} V\left(d_{1 j_{1}}, \ldots, d_{s j_{s}}\right) \geq|M| \\
& \geq \prod_{i=1}^{s} m_{i} \sum_{\substack{1 \leq j_{j} \leq b_{i} \\
1 \leq i \leq s}} V\left(d_{1 j_{1}}, \ldots, d_{s j_{s}}\right) .
\end{aligned}
$$

Next we observe that

$$
\begin{aligned}
|W| & =\sum_{\substack{1 \leq j_{i} \leq b_{i} \\
1 \leq i \leq s}} V\left(d_{1 j_{1}}, \ldots, d_{s j_{s}}\right) \\
& =\left|\begin{array}{ccccc}
b_{1} & b_{2} & \cdots & b_{s-1} & b_{s} \\
\sum_{j=1}^{b_{1}} d_{1 j} & \sum_{j=1}^{b_{2}} d_{2 j_{2}} & \cdots & \sum_{j=1}^{b s-1} d_{s-1 j_{s-1}} & \sum_{j=1}^{b_{s}} d_{s j_{s}} \\
\vdots & \vdots & \vdots & \vdots & \vdots \\
\sum_{j=1}^{b_{1}} d_{1 j_{1}}^{s-1} & \sum_{j=1}^{b_{2}} d_{2 j_{2}}^{s-1} & \cdots & \sum_{j=1}^{b_{s-1}} d_{s-1 j_{s-1}}^{s-1} & \sum_{j=1}^{b_{s}} d_{s j_{s}}^{s-1}
\end{array}\right| .
\end{aligned}
$$

Now by replacing the last column by the alternating sums of the columns, the last column becomes the transpose of the vector $\left((-1)^{s+1}, 0, \ldots, 0\right)$. Expanding the determinant along the last column, we get $|W|=|N|$. Since at least one of the Vandermonde determinants in the sum for $|W|$ is positive, we conclude that $|N|>0$. Hence the desired result follows from (1) and (3).

Remark 1.3. It is clear from the proof of 1.2 that the conjectured bounds for the multiplicity follow already once the shifts in the resolution satisfy the following condition (which is obviously weaker than quasi-purity):

$$
V\left(d_{1 j_{1}}, \ldots, d_{s j_{s}}\right) \geq 0
$$

for all sequences $d_{1 j_{1}}, \ldots, d_{s j_{s}}$.

As an easy consequence of 1.2 , we get

Corollary 1.4 (Huneke-Miller). Suppose that $S$ is Cohen-Macaulay with a pure resolution

$$
0 \rightarrow R^{b_{s}}\left(-d_{s}\right) \rightarrow \cdots \rightarrow R^{b_{2}}\left(-d_{2}\right) \rightarrow R^{b_{1}}\left(-d_{1}\right) \rightarrow R \rightarrow 0 .
$$

Then $e(S)=\prod_{i=1}^{n} d_{i} / s$ !.

When $S$ is not Cohen-Macaulay, the lower bound fails, as the next results show. Nevertheless we can establish the upper bound in the following cases. 
We first consider the case when the codimension of $S$ is one more than the projective dimension, in which case $S$ is called almost Cohen-Macaulay.

Theorem 1.5. Suppose that $S$ is almost Cohen-Macaulay of codimension $s-1$ and has a quasi-pure resolution. Then

$$
e(S) \leq 1 /(s-1) !\left(\prod_{i=1}^{s-1} M_{i}\right) .
$$

If the resolution of $S$ is pure, then

$$
e(S)=\left(\prod_{i=1}^{s-1} d_{i}\right) /(s-1) !-b_{s} \prod_{i=1}^{s-1}\left(\frac{d_{s}-d_{i}}{d_{i}}\right) /(s-1) ! .
$$

In particular, Conjecture 2 holds.

Proof. The proof proceeds along the same lines as in the Cohen-Macaulay case for the pure resolution and for the quasi-pure resolutions.

Here we consider the square matrix

$$
M=\left(\begin{array}{ccccc}
\sum_{k=1}^{b_{1}} 1 & \sum_{k=1}^{b_{2}} 1 & \cdots & \sum_{k=1}^{b_{s-1}} 1 & \sum_{k 1}^{b_{s}} 1 \\
\sum_{k=1}^{b_{1}} d_{1 k} & \sum_{k=1}^{b_{2}} d_{2 k} & \cdots & \sum_{k=1}^{b_{s-1}} d_{(s-1) k} & \sum_{k 1}^{b_{s}} d_{s k} \\
\sum_{k=1}^{b_{1}} d_{1 k}^{2} & \sum_{k=1}^{b_{2}} d_{2 k}^{2} & \cdots & \sum_{k=1}^{b_{s-1}} d_{(s-1) k}^{2} & \sum_{k 1}^{b_{s}} d_{s k}^{2} \\
\vdots & \vdots & \vdots & \vdots & \vdots \\
\sum_{k=1}^{b_{1}} d_{1 k}^{s-1} & \sum_{k=1}^{b_{2}} d_{2 k}^{s-1} & \cdots & \sum_{k=1}^{b_{s-1}} d_{(s-1) k}^{s-1} & \sum_{k 1}^{b_{s}} d_{s k}^{s-1}
\end{array}\right)
$$

of size $s$ where $s-1=\operatorname{codim} S$.

Similar arguments as in the proof of 1.2 show that

$$
\begin{aligned}
|M| & =-(s-1) ! e|N|+\sum_{\substack{1 \leq j_{i} \leq b_{i} \\
1 \leq i \leq s}} \prod_{i=1}^{s-1} d_{i j_{i}} V\left(d_{1 j_{1}}, \ldots, d_{s-1 j_{s-1}}\right) \\
& \leq-(s-1) ! e|N|+\left(\prod_{i=1}^{s-1} M_{i}\right)|N|
\end{aligned}
$$

where

$$
N=\left(\begin{array}{cccc}
\sum_{j=1}^{b_{1}} d_{1 j} & \sum_{j=1}^{b_{2}} d_{2 j} & \cdots & \sum_{j=1}^{b_{s-1}} d_{s-1 j} \\
\sum_{j=1}^{b_{1}} d_{1 j}^{2} & \sum_{j=1}^{b_{2}} d_{2 j}^{2} & \cdots & \sum_{j=1}^{b_{s-1}} d_{s-1 j}^{2} \\
\vdots & \vdots & \vdots & \vdots \\
\sum_{j=1}^{b_{1}} d_{1 j}^{s-1} & \sum_{j=1}^{b_{2}} d_{2 j}^{s-1} & \cdots & \sum_{j=1}^{b_{s-1}} d_{s-1 j}^{s-1}
\end{array}\right) .
$$

Since the determinant of $M$ is positive, we have

$$
e(S)<\left(\prod_{i=1}^{s-1} M_{i}\right) /(s-1) !
$$

In particular, if the resolution is pure of length one more than the codimension of $S$, then

$$
e(S)=\left(\prod_{i=1}^{s-1} d_{i}\right) /(s-1) !-b_{s} \prod_{i=1}^{s-1}\left(d_{s}-d_{i}\right) /(s-1) !
$$


The next result requires no Cohen-Macaulay hypothesis at all. Instead we pose a linearity condition.

Theorem 1.6. Suppose that $S$ has a p-linear resolution. Then Conjecture 2 holds.

Proof. Without loss of generality we may assume that the base field $K$ is infinite. Since $S$ has a $p$-linear resolution it follows that any generic sequence $\mathbf{y}=y_{1}, \ldots, y_{n}$ of forms of degree 1 generating the maximal ideal $\mathfrak{n}$ of $S$ is a proper sequence. In other words, $\mathfrak{n}$ annihilates the kernel of

$$
S /\left(y_{1}, \ldots, y_{i-1}\right) S \stackrel{y_{i}}{\longrightarrow} S /\left(y_{1}, \ldots, y_{i-1}\right) S
$$

for $i=1, \ldots, n-1$; see Theorem 5.7 and its proof in [11].

Let $d=\operatorname{dim} S$; then, since the sequence $y$ is generic, the partial sequence $y_{1}, \ldots, y_{d}$ is a generic system of parameters, and we have $e(S) \leq \ell\left(S /\left(y_{1}, \ldots, y_{d}\right) S\right)$.

Let $\mathbf{z}=z_{1}, \ldots, z_{d}$ be elements of degree 1 in $R$ such that $y_{i}=z_{i}+I$ for $i=1, \ldots, d$. We set $\bar{R}=R / \mathbf{z} R, \bar{S}=S /\left(y_{1}, \ldots, y_{d}\right) S$ and $\bar{I}=I \bar{R}$. Then $\bar{R}$ is a polynomial ring of dimension $n-d$, and $\bar{S}=\bar{R} / \bar{I}$.

Denote by $\overline{\mathfrak{m}}$ the graded maximal ideal of $\bar{R}$. We claim that $\bar{I}=\overline{\mathfrak{m}}^{p}$. Indeed, suppose this is not the case. Then there exists an element $a \in \bar{S}$ of degree $t \geq p$ which belongs to the socle of $\bar{S}$.

Notice that $a$ belongs to the kernel of the map $\bar{S} \stackrel{y_{d+1}}{\rightarrow} \bar{S}$. From the long exact sequence of Koszul homology

$$
\cdots \longrightarrow H_{1}\left(y_{1}, \ldots, y_{d+1} ; S\right) \longrightarrow \bar{S}(-1) \stackrel{y_{d+1}}{\longrightarrow} \bar{S} \longrightarrow \bar{S} / y_{d+1} \bar{S} \longrightarrow 0
$$

we then deduce that the homogeneous component $H_{1}\left(y_{1}, \ldots, y_{d+1} ; S\right)_{t+1}$ is nonzero.

Since $y_{1}, \ldots, y_{n}$ is a proper sequence it follows that the natural map

$$
H_{1}\left(y_{1}, \ldots, y_{d+1} ; S\right) \longrightarrow H_{1}\left(y_{1}, \ldots, y_{n} ; S\right)
$$

is injective; see [11]. Therefore, $H_{1}\left(y_{1}, \ldots, y_{n} ; S\right)$ has a nontrivial homogeneous component of degree $>p$. This is a contradiction since $H_{1}\left(y_{1}, \ldots, y_{n} ; S\right)$ is isomorphic to $\operatorname{Tor}_{1}^{R}(S ; K)$, and since $\operatorname{Tor}_{1}^{R}(S ; K)$ is a graded $K$-vector space concentrated in degree $p$.

Now, as we know that $\bar{I}=\overline{\mathfrak{m}}^{p}$, we get

$$
e(S) \leq \ell\left(\bar{R} / \overline{\mathfrak{m}}^{p}\right)=\left(\begin{array}{c}
n-d+p-1 \\
n-d
\end{array}\right)=\left(\begin{array}{c}
h+p-1 \\
h
\end{array}\right) .
$$

Another interesting situation is when $S$ is Cohen-Macaulay with a resolution which may not be quasi-pure, but essentially so. To be precise, we consider the multisets (sets with repetitions of elements)

$$
D_{i}=\left\{d_{i 1}, \ldots, d_{i b_{i}}\right\}, \quad i=1, \ldots, s .
$$

Then the left hand side of the formulas in 1.1 may be written as

$$
\sum_{i=1}^{s} \sum_{a \in D_{i}}(-1)^{i} a^{k}
$$

It is clear that these sums remain unchanged if we move an element from $D_{i}$ to $D_{i+2 k}$ for some $k$. We say that a resolution is numerically equivalent to a virtual resolution with the collection of multisets $\left\{D_{i}^{\prime}: 1 \leq i \leq t\right\}$, if after a finite number of such movements, the multisets $\left\{D_{i}, 1 \leq i \leq s\right\}$ of the resolution become the 
multisets $\left\{D_{i}^{\prime}, 1 \leq i \leq t\right\}$. Of course, the total number of multisets may not be the same. We call a resolution numerically quasi-pure, if after a finite number of such operations the new multisets $D_{1}^{\prime}, \ldots, D_{s}^{\prime}$ satisfy the quasi-pure condition, that is, any element in $D_{i}^{\prime}$ is greater than or equal to any element in $D_{i-1}^{\prime}$ for all $i$. Thus 1.2 yields

Corollary 1.7. Let $S$ be Cohen-Macaulay with a numerically quasi-pure resolution, and let the $D_{i}^{\prime}$ be defined as above. We set $M_{i}^{\prime}=\max \left\{a: a \in D_{i}\right\}$, and $m_{i}^{\prime}=\min \left\{a: a \in D_{i}^{\prime}\right\}$. Then

$$
\left(\prod_{i=1}^{s} m_{i}^{\prime}\right) / s ! \leq e(S) \leq\left(\prod_{i=1}^{s} M_{i}^{\prime}\right) / s !
$$

We apply this idea of numerical equivalence in the proof of the next theorem, in which we consider the case where all but one of the maps in the resolution are in pure degree $d_{i}$.

Theorem 1.8. Suppose that $S$ is Cohen-Macaulay with a minimal graded resolution

$$
\begin{aligned}
0 \longrightarrow R^{b_{s}}\left(-d_{s}\right) \longrightarrow & \cdots \longrightarrow \bigoplus_{j=1}^{b_{t}} R\left(-d_{t j}\right) \longrightarrow \\
& \cdots \longrightarrow R^{b_{1}}\left(-d_{1}\right) \longrightarrow R \longrightarrow 0,
\end{aligned}
$$

so that the shifts are in pure degree $d_{i}$, except possibly at the th place for some $t$, $1 \leq t \leq s$. Then the upper bound of Conjecture 1 holds.

Proof. Without loss of generality, $d_{t 1} \leq d_{t 2} \leq \cdots \leq d_{t b_{t}}$. By 1.2 , we may assume that the resolution is not quasi-pure. Then there exists an integer $q, 1 \leq q \leq s-t$, such that for all integers $p, 1 \leq p \leq q$, there exists an integer $k_{p}$ such that

$$
d_{t+p} \geq d_{t j} \quad \text { for } j<k_{p}, \quad \text { and } \quad d_{t+p}<d_{t k_{p}} .
$$

For convenience, we also set $k_{0}=1$.

Now we move the elements $d_{t j}, k_{2 p-1} \leq j \leq k_{2 p}-1$ to $D_{t+2 p}$ for all $p$ with $0 \leq 2 p \leq q$. In other words, the resolution of $S$ is numerically equivalent to a virtual resolution,

$$
\begin{aligned}
& \cdots \longrightarrow R^{b_{t+2 p}}\left(-d_{t+2 p}\right) \oplus \bigoplus_{j=k_{2 p-1}}^{k_{2 p}-1} R\left(-d_{t j}\right) \longrightarrow \cdots \\
& \cdots \quad \longrightarrow \bigoplus_{0 \leq p \leq q / 2} \bigoplus_{j=k_{2 p}}^{k_{2 p+1}-1} R\left(-d_{t j}\right) \oplus \bigoplus_{j=k_{q}}^{b_{t}} R\left(-d_{t j}\right) \longrightarrow \cdots \\
& \cdots \quad \longrightarrow R^{b_{1}}\left(-d_{1}\right) \longrightarrow R \longrightarrow 0
\end{aligned}
$$

Now we consider a matrix $M$ as in the proof of 1.2 for the multisets corresponding to these virtual resolutions. The Vandermonde determinants that occur in the sum are nonnegative because, in every such determinant, there will be an even number of $d_{i j_{i}}$ which are less than any given $d_{t j_{t}}$. So, we may proceed as in the proof of 1.2 . Furthermore, we have modified our multisets while maintaining all the $M_{i}$ except for $M_{t}$ which is actually lower. So, we get the result. 


\section{LOW CODIMENSION}

In this section we consider codimension 2 perfect ideals, and codimension 3 Gorenstein ideals.

To begin with, we consider a perfect graded ideal $I \subset R$ of codimension 2 in the polynomial ring $R=K\left[x_{1}, \ldots, x_{n}\right]$. Let $a_{1} \leq a_{2} \leq \ldots \leq a_{m}$ be the degrees of a mimimal set of generators of $I$. Then $I$ has a minimal free resolution of the form

$$
0 \longrightarrow \bigoplus_{i=1}^{m-1} R\left(-b_{i}\right) \longrightarrow \bigoplus_{i=1}^{m} R\left(-a_{i}\right) \longrightarrow I \longrightarrow 0
$$

We may assume that $b_{1} \leq \ldots \leq b_{m-1}$. We set $u_{i j}=b_{i}-a_{j}$ for all $i$ and $j$, and call $\left(u_{i j}\right)$ the degree matrix of $I$. If $\left(g_{i j}\right)$ is the relation matrix of $I$, then of course $u_{i j}=\operatorname{deg} g_{i j}$ for all $i$ and $j$ with $g_{i j} \neq 0$.

We now quote a few results from [12] which will be used in the proof of the next theorem: set $u_{i}=u_{i i}$ and $v_{i}=u_{i i+1}$; then one has

(a) $u_{i} \geq v_{i} \geq 0$ for $i=1, \ldots, m-1$;

(b) $u_{i+1} \geq v_{i}$ for $i=1, \ldots, m-2$;

(c) $a_{1}=v_{1}+\cdots+v_{m-1}$ and $a_{m}=u_{1}+\cdots+u_{m-1}$;

(d) $b_{1}=v_{1}+\cdots+v_{m-1}+u_{1}$ and $b_{m-1}=u_{1}+\cdots+u_{m-1}+v_{m-1}$;

(e) $e(R / I)=\sum_{i=1}^{m-1} u_{i}\left(v_{i}+\cdots+v_{m-1}\right)$.

Observe that the $u_{i}$ and $v_{i}$ determine the whole degree matrix, and conversely any two sequences $u_{i}, v_{i}$ satisfying (a) and (b) arise from a graded perfect ideal of codimension 2 .

Theorem 2.1. Let $I \subset R$ be a graded perfect ideal of codimension 2. With the notation introduced we have

$$
a_{1} b_{1} \leq 2 e(R / I) \leq a_{m} b_{m-1} .
$$

Proof. We first prove the lower inequality: we want to show that

$$
\sum_{i=1}^{m-1} 2 u_{i}\left(v_{i}+\cdots+v_{m-1}\right) \geq\left(v_{1}+\cdots+v_{m-1}\right)\left(u_{1}+v_{1}+\cdots+v_{m-1}\right) .
$$

Observing that

$$
2 u_{1}\left(v_{1}+\cdots+v_{m-1}\right) \geq u_{1}\left(v_{1}+\cdots+v_{m-1}\right)+v_{1}\left(v_{1}+\cdots+v_{m-1}\right),
$$

and that $2 u_{i} \geq\left(v_{i-1}+v_{i}\right)$ for $i=2 \ldots, m-1$, it remains to show that

$$
\begin{aligned}
& \sum_{i=2}^{m-1}\left(v_{i-1}+v_{i}\right)\left(v_{i}+\cdots+v_{m-1}\right) \\
& \quad=\left(v_{1}+\cdots+v_{m-1}\right)\left(v_{2}+\cdots+v_{m-1}\right) .
\end{aligned}
$$

The right hand side of (4) may be written as

$$
\left(v_{1}+v_{2}\right)\left(v_{2}+\cdots+v_{m-1}\right)+\left(v_{2}+\cdots+v_{m-1}\right)\left(v_{3}+\cdots+v_{m-1}\right) .
$$

The first of this summands cancels against the first summand on the left of (4), and one obtains

$$
\sum_{i=3}^{m-1}\left(v_{i-1}+v_{i}\right)\left(v_{i}+\cdots+v_{m-1}\right)=\left(v_{2}+\cdots+v_{m-1}\right)\left(v_{3}+\cdots+v_{m-1}\right) .
$$

Hence the assertion follows by induction. 
In order to prove the upper inequality we have to show that

$$
\left(u_{1}+\cdots+u_{m-1}\right)\left(u_{1}+\cdots+u_{m-1}+v_{m-1}\right) \geq \sum_{i=1}^{m-1} 2 v_{i}\left(u_{1}+\cdots+u_{i}\right) .
$$

Here we used

$$
\sum_{i=1}^{m-1} 2 u_{i}\left(v_{i}+\cdots+v_{m-1}\right)=\sum_{i=1}^{m-1} 2 v_{i}\left(u_{1}+\cdots+u_{i}\right)
$$

Observing that

$$
2 v_{m-1}\left(u_{1}+\cdots+u_{m-1}\right) \leq v_{m-1}\left(u_{1}+\cdots+u_{m-1}\right)+u_{m-1}\left(u_{1}+\cdots+u_{m-1}\right),
$$

and that $2 v_{i} \leq\left(u_{i}+u_{i+1}\right)$ for $i=1, \ldots, m-2$, it remains to show

$$
\begin{aligned}
& \sum_{i=1}^{m-2}\left(u_{1}+\cdots+u_{i}\right)\left(u_{i}+u_{i+1}\right) \\
& \quad=\left(u_{1}+\cdots+u_{m-1}\right)\left(u_{1}+\cdots+u_{m-2}\right) .
\end{aligned}
$$

The right hand side of (5) may be written as

$$
\left(u_{m-2}+u_{m-1}\right)\left(u_{1}+\cdots+u_{m-2}\right)+\left(u_{1}+\cdots+u_{m-2}\right)\left(u_{1}+\cdots+u_{m-3}\right)
$$

The first of this summands cancels against the last summand on the left of (5), and one obtains

$$
\sum_{i=1}^{m-3}\left(u_{1}+\cdots+u_{i}\right)\left(u_{i}+u_{i+1}\right)=\left(u_{1}+\cdots+u_{m-2}\right)\left(u_{1}+\cdots+u_{m-3}\right) .
$$

Hence the assertion follows by induction.

Among the perfect ideals of codimension 3, the Gorenstein ideals are best understood. For the rest of this section we discuss our conjecture for this class of ideals.

The Buchsbaum-Eisenbud structure theorem [3] identifies them as ideals of pfaffians. For monomial Gorenstein ideals of codimension 3 one has an even more restrictive structure theorem; see [5]. We shall use these results in our computations.

To describe the results we fix some notation. Let $I$ be a graded Gorenstein ideal of codimension 3 in $R=K\left[x_{1}, \ldots, x_{n}\right]$. As a consequence of the BuchsbaumEisenbud structure theorem, $I$ is generated by an odd number of homogeneous generators, say $y_{1}, \ldots, y_{2 r+1}$. Let $a_{i}$ be the degree of $y_{i}$ for $i=1, \ldots, 2 r+1$. We may assume that $a_{1} \leq a_{2} \leq \ldots \leq a_{2 r+1}$. Let $c$ be the last shift in the resolution. Then $R / I$ has a resolution of the form

$$
0 \rightarrow R(-c) \rightarrow \bigoplus_{j=1}^{2 r+1} R\left(-\left(c-a_{j}\right)\right) \rightarrow \bigoplus_{j=1}^{2 r+1} R\left(-a_{j}\right) \rightarrow R \rightarrow 0,
$$

and our conjectured inequalities are

$$
c a_{1}\left(c-a_{2 r+1}\right) \leq 6 e(R / I) \leq c a_{2 r+1}\left(c-a_{1}\right)
$$

Here we show that the conjectured upper bound holds. We also prove the lower bound in the following cases:

(a) $c \geq 2 a_{2 r+1}$,

(b) $I$ is generated by 5 elements, 
(c) $I$ is a monomial ideal and $a_{1}=r$.

Theorem 2.2. Let $I \subset R$ be a graded Gorenstein ideal of codimension 3 generated by $2 r+1$ elements. With the notation introduced, assume that $c \geq 2 a_{2 r+1}$. Then Conjecture 1 holds.

Proof. By 1.1, we get $6 e(R / I)=c^{3}-\sum_{i=1}^{2 r+1}\left(\left(c-a_{i}\right)^{3}-a_{i}^{3}\right)$ We note also that $r c=\sum_{i=1}^{2 r+1} a_{i}$. So, we get that

$$
6 e(R / I)=c^{3}(-2 r)-c^{2}\left(3 \sum_{i=1}^{2 r+1} a_{i}\right)+3 c\left(\sum_{i=1}^{2 r+1} a_{i}^{2}\right)+2 \sum_{i=1}^{2 r+1} a_{i}^{3} .
$$

This simplifies to

$$
\begin{aligned}
6 e(R / I) & =c^{2}(-2 r c)+c^{2}\left(3 \sum_{i=1}^{2 r+1} a_{i}\right)-3 c\left(\sum_{i=1}^{2 r+1} a_{i}^{2}\right)+2 \sum_{i=1}^{2 r+1} a_{i}^{3} \\
& =c^{2}\left(\sum_{i=1}^{2 r+1} a_{i}\right)-3 c\left(\sum_{i=1}^{2 r+1} a_{i}^{2}\right)+2 \sum_{i=1}^{2 r+1} a_{i}^{3} \\
& =\sum_{i=1}^{2 r+1}\left(a_{i}\left(c^{2}-3 c a_{i}+2 a_{i}^{2}\right)\right) \\
& =\sum_{i=1}^{2 r+1} a_{i}\left(c-a_{i}\right)\left(c-2 a_{i}\right) .
\end{aligned}
$$

If $c \geq 2 a_{2 r+1}$, it follows that

$$
a_{1}\left(c-a_{2 r+1}\right) \sum_{j=1}^{2 r+1}\left(c-2 a_{j}\right) \leq 6 e \leq a_{2 r+1}\left(c-a_{1}\right) \sum_{j=1}^{2 r+1}\left(c-2 a_{j}\right) .
$$

Since $\sum_{j=1}^{2 r+1}\left(c-2 a_{j}\right)=c$, the conclusion follows.

The conjectured upper bound for the multiplicity will follow from the next numerical result.

Lemma 2.3. Given integers $a_{1}, \ldots, a_{m}, m=2 r+1$, and an integer $c$ such that

(1) $a_{1} \leq a_{2} \leq \ldots \leq a_{m}$;

(2) $\sum_{i=1}^{m} a_{i}=r c$;

(3) $c \geq a_{i}+a_{m+2-i}$ for $i \geq 2$.

Then $c a_{m}\left(c-a_{1}\right) \geq \sum_{i=1}^{m} a_{i}\left(c-a_{i}\right)\left(c-2 a_{i}\right)$.

Proof. If $c \geq 2 a_{m}$, then we have the result by the same arguments as in the proof of 2.2. So we may assume that $c<2 a_{m}$.

We set $M=c a_{m}\left(c-a_{1}\right)-\sum_{i=1}^{2 r+1} a_{i}\left(c-a_{i}\right)\left(c-2 a_{i}\right)$. We must show that $M \geq 0$.

Let $t$ be such that $c \geq 2 a_{t}$ and $c \leq 2 a_{t+1}$. Then, $t \geq r+1$. Since

$$
a_{i}\left(c-a_{i}\right)-a_{j}\left(c-a_{j}\right)=\left(a_{i}-a_{j}\right)\left(c-a_{i}-a_{j}\right),
$$

it follows that

$$
a_{i}\left(c-a_{i}\right) \leq a_{t}\left(c-a_{t}\right) \quad \text { for all } \quad i \leq t
$$

and

$$
a_{i}\left(c-a_{i}\right) \geq a_{m}\left(c-a_{m}\right) \text { for all } i \geq t+1 .
$$


Therefore,

$$
\begin{aligned}
M & =a_{m} c\left(c-a_{1}\right)-\sum_{i=t+1}^{m} a_{i}\left(c-a_{i}\right)\left(c-2 a_{i}\right)-\sum_{i=1}^{t} a_{i}\left(c-a_{i}\right)\left(c-2 a_{i}\right) \\
& \geq a_{m} c\left(c-a_{1}\right)+a_{m}\left(c-a_{m}\right) \sum_{i=t+1}^{m}\left(2 a_{i}-c\right)-\sum_{i=1}^{t} a_{i}\left(c-a_{i}\right)\left(c-2 a_{i}\right) \\
& =a_{m}\left[c\left(c-a_{1}\right)+\left(c-a_{m}\right) \sum_{i=t+1}^{m}\left(c-2 a_{i}\right)\right]-\sum_{i=1}^{t} a_{i}\left(c-a_{i}\right)\left(c-2 a_{i}\right) \\
& =a_{m} c\left(a_{m}-a_{1}\right)+\sum_{i=1}^{t}\left(a_{m}\left(c-a_{m}\right)-a_{i}\left(c-a_{i}\right)\right)\left(c-2 a_{i}\right) \\
& =a_{m} c\left(a_{m}-a_{1}\right)+\sum_{i=1}^{t}\left(a_{m}-a_{i}\right)\left(c-a_{m}-a_{i}\right)\left(c-2 a_{i}\right)
\end{aligned}
$$

Thus the lemma is proved if $c \geq a_{m}+a_{t}$. If not, $c<a_{m}+a_{t}$, and we distinguish two cases:

(a) $a_{1} \leq \ldots \leq a_{t}<a_{t+1} \leq \ldots \leq a_{k}<a_{k+1}=\ldots=a_{m}$.

(b) $a_{1} \leq \ldots \leq a_{t}<a_{t+1} \leq a_{t+2}=\ldots=a_{m}$.

Case (a): We reduce to case (b). Let $s \geq t$ be the smallest integer for which there exists a $k>s$ such that $c-a_{s}<a_{k}<a_{m}$. When such an $s$ does not exist, we are in case (b). We choose $k$ to be the largest such integer.

If $c=a_{k}+a_{2 r+3-k}$, then

$$
a_{k}\left(c-a_{k}\right)\left(2 a_{k}-c\right)=a_{2 r+3-k}\left(c-a_{2 r+3-k}\right)\left(c-2 a_{2 r+3-k}\right) .
$$

Hence, we can remove both $a_{k}$ and $a_{2 r+3-k}$ altering neither $M$ nor any of our hypotheses and have $M \geq 0$ by induction on $r$. (The case $r=0$ is trivial.) So, we may assume that $c>a_{k}+a_{2 r+3-k}$. Now, if we replace $a_{k}$ by $a_{k}+1$ and $a_{i}$ by $a_{t}-1$ where $i$ is the smallest index for which $a_{i}=a_{t}$, it does not alter any of our hypotheses and decreases $M$ by $6\left(a_{k}-a_{i}+1\right)\left(a_{k}+a_{i}-c\right)$. We repeat this process. After a finite number of steps we are in case 2, or we are done because $c \geq a_{t}+a_{m}$.

Case (b): By (6), $M \geq a_{m} c\left(a_{m}-a_{1}\right)+\sum_{i=1}^{t}\left(a_{m}-a_{i}\right)\left(c-a_{m}-a_{i}\right)\left(c-2 a_{i}\right)$. By hypothesis, $c \geq a_{m}+a_{m+2-t-2}=a_{m}+a_{m-t}$, and so,

$$
\sum_{i=1}^{m-t}\left(a_{m}-a_{i}\right)\left(c-a_{m}-a_{i}\right)\left(c-2 a_{i}\right) \geq 0
$$

Thus we get

$$
M \geq a_{m} c\left(a_{m}-a_{1}\right)-\sum_{i=m-t+1}^{t}\left(a_{m}-a_{i}\right)\left(a_{m}+a_{i}-c\right)\left(c-2 a_{i}\right) .
$$


Since $a_{m} \geq a_{m}+a_{i}-c$ and $a_{m}-a_{1} \geq a_{m}-a_{i}$, we just need to check that $c \geq \sum_{i=m-t+1}^{t}\left(c-2 a_{i}\right)$, equivalently, $\sum_{i=m-t+1}^{t} 2 a_{i} \geq(2 t-m-1) c$. But,

$$
\begin{aligned}
r c & =\sum_{i=2}^{m-t}\left(a_{i}+a_{m}\right)+\left(a_{1}+a_{t+1}\right)+\sum_{i=m-t+1}^{t} a_{i} \\
& \leq(m-t) c+\sum_{i=m-t+1}^{t} a_{i} .
\end{aligned}
$$

Therefore $(r+t-m) c \leq \sum_{i=m-t+1}^{t} a_{i}$, and hence

$$
(2 t-m-1) c=(2 r+2 t-2 m) c \leq 2 \sum_{i=m-t+1}^{t} a_{i},
$$

as desired.

Theorem 2.4. Let $I \subset R$ be a graded Gorenstein ideal of codimension 3 as above. Then the conjectured upper bound holds for the multiplicity of $R / I$.

Proof. Let $a_{i}, i=1,2, \ldots, 2 r+1=m$, be the degrees of the generators of $I$ in ascending order. So that, $r c=\sum_{i=1}^{2 r+1} a_{i}$ and $c \geq a_{i}+a_{2 r+3-i}$ for all i.

By 2.3 we have

$$
a_{m}\left(c-a_{1}\right) c \geq \sum_{i=1}^{2 r+1} a_{i}\left(c-a_{i}\right)\left(c-2 a_{i}\right)=6 e(R / I) .
$$

Theorem 2.5. Let $I \subset R$ be as above a graded Gorenstein ideal of codimension 3 generated by 5 elements. Then the conjectured lower bound holds for the multiplicity of $R / I$.

Proof. We need to prove the inequality $a_{1}\left(c-a_{5}\right) c \leq 6 e$. By 2.2, we may assume that $c<2 a_{5}$. We will use the formula of Herzog, Trung and Valla [12] for the multiplicity of a graded Gorenstein ideal of codimension 3. In our particular case the formula is

$$
e=v_{1}\left(u_{1}+u_{2}\right)\left(w_{1}+w_{2}\right)+v_{2} u_{2} w_{2}
$$

where $v_{i}=c-a_{i}-a_{6-i}, w_{i}=c-a_{i+1}-a_{6-i}$ and $u_{i}=c-a_{i}-a_{5-i}$ for $i=1,2$. So, $u_{1}+u_{2}=a_{5}$ and $w_{1}+w_{2}=a_{1}$. It follows that $e=v_{1} a_{5} a_{1}+v_{2} u_{2} w_{2}$.

We will distinguish two cases. First we assume that $w_{2} \leq v_{1}$. Then

$$
\begin{aligned}
c a_{1}\left(c-a_{5}\right) & =c a_{1}\left(v_{1}+a_{1}\right)=c a_{1} v_{1}+c a_{1}\left(w_{1}+w_{2}\right) \\
& <2 a_{5} a_{1} v_{1}+2 a_{5} a_{1} v_{1}+2 a_{5} a_{1} v_{1}<6 e .
\end{aligned}
$$

In the second case we assume that $w_{2}>v_{1}$. Then $a_{1}+a_{5}>a_{3}+a_{4}$, and hence

$$
\begin{aligned}
2 u_{2}-u_{1} & =2 c-2 a_{2}-2 a_{3}-c+a_{4}+a_{1} \\
& =\left(c-a_{2}-a_{5}\right)+\left(a_{5}-a_{3}\right)+\left(a_{4}-a_{3}\right)+a_{1}-a_{2} \\
& >a_{4}-a_{1}+a_{1}-a_{2} \geq 0,
\end{aligned}
$$

which implies $a_{5}=u_{1}+u_{2}<3 u_{2}$. 
Now we get

$$
\begin{aligned}
c a_{1}\left(c-a_{5}\right) & =c a_{1}\left(v_{1}+a_{1}\right) \leq 2 a_{5} v_{1} a_{1}+2 a_{5} a_{1} w_{1}+2 a_{5} a_{1} w_{2} \\
& =2 a_{5} v_{1} a_{1}+2 a_{5} w_{1} a_{1}+2 a_{5} w_{1} w_{2}+2 a_{5} w_{2} w_{2} \\
& <2 a_{5} v_{1} a_{1}+2 a_{5} v_{1} a_{1}+2 a_{5} v_{1} a_{1}+6 u_{2} v_{2} w_{2}=6 e .
\end{aligned}
$$

We now turn to the monomial Gorenstein ideals of codimension 3. By the structure theorem [5] for monomial Gorenstein ideals of codimension 3, there exist relatively prime monomials $p_{1}, \ldots, p_{2 r+1}$ such that $R / I$ is isomorphic to $R / J$ where $J$ is minimally generated by the monomials,

$$
\left\{\prod_{i=t}^{r+t-1} p_{i}: 1 \leq t \leq 2 r+1, \quad p_{2 r+1+i}=p_{i}\right\}
$$

Let $a_{i}=\operatorname{deg}\left(p_{i}\right)$. Then the graded resolution of $R / J$ is of the form

$$
0 \rightarrow R\left(-\sum_{i=1}^{2 r+1} a_{i}\right) \rightarrow \bigoplus_{t=1}^{2 r+1} R\left(-\sum_{i=t}^{r+t} a_{i}\right) \rightarrow \bigoplus_{t=1}^{2 r+1} R\left(-\sum_{i=t}^{r+t-1} a_{i}\right) \rightarrow R \rightarrow 0
$$

With this notation we have

Lemma 2.6. Let $I$ be a Gorenstein ideal of codimension 3 generated by $2 r+1$ monomials. Then $3 e(R / I)=\sum_{i=1}^{m} a_{i} T_{i}$, and

$$
T_{i}+T_{r+i+1}=\left(\sum_{k=i+1}^{r+i} a_{k}\right)\left(\sum_{k=r+i+1}^{2 r+1+i} a_{k}\right)
$$

where we set $a_{t}=a_{t+k(2 r+1)}$, for all integers $k$ and $t$.

Proof. By 1.1, we get

$$
6(R / I)=\left(\sum_{t=1}^{2 r+1} a_{i}\right)^{3}-\sum_{t=1}^{2 r+1}\left(\left(\sum_{i=t}^{r+t} a_{i}\right)^{3}-\left(\sum_{i=t}^{r+t-1} a_{i}\right)^{3}\right)
$$

This expression is symmetric in $a_{j}$ for all $j$. Let $T_{j}$ be the coefficient of $a_{j}$ in the expression for the multiplicity $e(R / I)$, so that, $e=a_{j} T_{j}+D$ where $D$ is independent of $a_{j}$. In the light of the symmetry observed above, it suffices to prove that:

(a) There are no terms containing $a_{1}^{2}$ or $a_{1}^{3}$ and

(b) $T_{1}+T_{r+2}=\left(\sum_{k=2}^{r+1} a_{k}\right)\left(\sum_{k=r+2}^{2 r+2} a_{k}\right)$.

So, we just pick terms with $a_{1}$ in

$$
\begin{aligned}
6(R / I) & =\left(\sum_{t=1}^{2 r+1} a_{i}\right)^{3}-\sum_{t=1}^{2 r+1}\left(\left(\sum_{i=t}^{r+t} a_{i}\right)^{3}-\left(\sum_{i=t}^{r+t-1} a_{i}\right)^{3}\right) \\
& =\left(\sum_{t=1}^{2 r+1} a_{i}\right)^{3}-\sum_{t=1}^{2 r+1} a_{r+t}\left(a_{r+t}^{2}+3 a_{r+t} \sum_{i=t}^{r+t-1} a_{i}+3\left(\sum_{i=t}^{r+t-1} a_{i}\right)^{2}\right) .
\end{aligned}
$$


The terms with $a_{1}$ are

$$
\begin{aligned}
3 a_{1}^{2}\left(\sum_{i=2}^{2 r+1} a_{i}\right) & +3 a_{1}\left(\sum_{i=2}^{2 r+1} a_{i}\right)^{2} \\
& -3 a_{1}\left(\sum_{i=r+2}^{2 r+1} a_{i}\right)^{2}-3 a_{1}^{2}\left(\sum_{i=2}^{2 r+1} a_{i}\right)-3 a_{1}\left(\sum_{i=2}^{r+1} a_{i}^{2}\right) \\
& -6 a_{1}\left(\sum_{j=2}^{r}\left(\sum_{i=r+j+1}^{2 r+1} a_{i}+\left(\sum_{i=2}^{j-1} a_{i}\right)\right) a_{j}\right)-6 a_{1} a_{r+1} \sum_{i=2}^{r} a_{i} \\
=6 a_{1} & \left(\sum_{j=3}^{r} a_{j} \sum_{i=2}^{j-1} a_{i}\right)+\left(\sum_{i=2}^{r+1} a_{i}\right)\left(\sum_{i=r+2}^{2 r+1} a_{i}\right) \\
& -\left(\sum_{j=2}^{r}\left(\sum_{i=r+j+1}^{2 r+1} a_{i}+\left(\sum_{i=2}^{j-1} a_{i}\right) a_{j}\right)\right]
\end{aligned}
$$

Hence we see that there are no terms with $a_{1}^{2}$ and that

$$
T_{1}=a_{2} a_{r+2}+a_{3}\left(a_{r+2}+a_{r+3}\right)+\cdots+a_{r+1}\left(\sum_{i=r+2}^{2 r+1} a_{i}\right) .
$$

So, by symmetry,

$$
T_{r+2}=a_{r+3} a_{2}+a_{r+4}\left(a_{2}+a_{3}\right)+\cdots+a_{1}\left(\sum_{i=2}^{r+1} a_{i}\right) .
$$

Thus,

$$
\begin{aligned}
T_{1}+T_{r+2}= & a_{2} a_{r+2}+a_{3}\left(a_{r+2}+a_{r+3}\right)+\cdots+a_{r+1}\left(\sum_{i=r+2}^{2 r+1} a_{i}\right) \\
& \quad+a_{r+3} a_{2}+a_{r+4}\left(a_{2}+a_{3}\right)+\cdots+a_{1}\left(\sum_{i=2}^{r+1} a_{i}\right) \\
= & \left(\sum_{i=2}^{r+1} a_{i}\right)\left(\sum_{i=r+2}^{2 r+2} a_{i}\right)
\end{aligned}
$$

This concludes the proof because by symmetry we get,

$$
3 e(R / I)=\sum_{j=1}^{2 r+1} a_{j} T_{j}
$$

and

$$
T_{i}+T_{r+i+1}=\left(\sum_{k=i+1}^{r+i} a_{k}\right)\left(\sum_{k=r+i+1}^{2 r+1+i} a_{k}\right)
$$

Next, we prove the lower bound on $e(R / I)$ if we assume that the smallest degree for the generator is $r$. 
Theorem 2.7. Let I be a grade three Gorenstein ideal generated by $2 r+1$ monomials. Then

$$
6 e(R / I) \geq r m_{2} m_{3} .
$$

In particular, if one of the generators has the smallest possible degree, namely $r$, then we have the conjectured lower bound.

Proof. By 2.6, the multiplicity is given by $6 e(R / I)=2 \sum_{i=1}^{m} a_{i} T_{i}$ where $T_{i}+$ $T_{r+i+1}=\left(\sum_{k=i+1}^{r+i} a_{k}\right)\left(\sum_{k=r+i+1}^{2 r+1+i} a_{k}\right)$. Hence,

$$
\begin{aligned}
6 e(R / I) & =\sum_{i=1}^{m}\left(a_{i} T_{i}+a_{r+i+1} T_{r+i+1}\right) \geq \sum_{i=1}^{n}\left[T_{i}+T_{r+i+1}\right] \\
& =\sum_{i=1}^{n}\left(\sum_{k=i+1}^{r+i} a_{k}\right)\left(\sum_{k=r+i+1}^{2 r+1+i} a_{k}\right) \geq m_{2} \sum_{i=1}^{n}\left(\sum_{k=i+1}^{r+i} a_{k}\right) \\
& =m_{2} r m_{3} .
\end{aligned}
$$

\section{Stable ideals}

In this section we prove our conjecture for stable ideals. We first introduce some notation: for a monomial $u \in R$ we let $m(u)=\max (u)$ be the largest integer such that $x_{m(u)}$ divides $u$, and $\min (u)$ the smallest such number. According to Eliahou and Kervaire one calls a monomial ideal $I$ stable, if for all monomials $u \in I$ one has $\left(x_{i} u\right) / x_{m(u)} \in I$ for all $i \leq m(u)$. The importance of this notion is partially due to the fact that the generic initial ideal of an arbitrary homogeneous ideal is stable (even strongly stable), if char $K=0$.

It suffices to check the property 'stable' for the generators of the monomial ideal $I$. A minimal set of monomial generators of $I$ is uniquely determined. We denote this set by $G(I)$. A subset $S$ of monomials of $G(I)$ with the property that any $u \in G(I)$ is of the form $\left(x_{i} v\right) / x_{m(v)}$ for some $v \in S$ and some $i \leq m(v)$ is called a set of Borel generators of $I$. We denote the unique smallest such set by $B(I)$.

As in the introduction, we let $M_{i}=M_{i}(I)$ be largest, and $m_{i}=m_{i}(I)$ be the smallest $i$ th shift in the resolution of $R / I$. It follows from the graded EliahouKervaire resolution $F$ of $R / I$ that

$$
M_{i}=\max \{\operatorname{deg} u: u \in G(I), m(u) \geq i\}+i-1
$$

and

$$
m_{i}=\min \{\operatorname{deg} u: u \in G(I), m(u) \geq i\}+i-1 .
$$

Indeed, $F_{i}$ is a free $R$-module with homogeneous basis $f(\sigma ; u)$ where $u \in G(I)$ and $\sigma \subset\{1, \ldots, n\}$ with $\max (\sigma)<\max (u)$ and $|\sigma|=i-1$. Moreover, $\operatorname{deg} f(\sigma ; u)=$ $\operatorname{deg} u+(i-1)$.

For later applications we need

Lemma 3.1. Let $I \subset R$ be a stable ideal. Then

(a) $\operatorname{codim} R / I=\max \{\min (u): u \in I\}=\max \{\min (u): u \in G(I)\}$;

(b) $\operatorname{codepth} R / I=\max \{\max (u): u \in G(I)\}$.

Proof. (a) The associated prime ideals containing $I$ are all of the form $\left(x_{1}, \ldots, x_{i}\right)$ for some $i$. Thus there is just one minimal prime, and obviously it is equal to $\left(x_{1}, \ldots, x_{j}\right)$ where $j=\max \{\min (u): u \in I\}$. This proves the first assertion. 
(b) follows immediately from the description of the resolution of $R / I$.

Our main result of this section is

Theorem 3.2. Let $I \subset R$ be a stable ideal such that $R / I$ is Cohen-Macaulay. Then Conjecture 1 holds.

The proof of the theorem is based on a few technical lemmata.

We first remark that in the above formulas for $m_{i}$ and $M_{i}$ we may replace $G(I)$ by $B(I)$, if we wish. We also may replace $m(u) \geq i$ by $m(u)=i$ in the second formula. Indeed we have

Lemma 3.3. $m_{i}=\min \{\operatorname{deg} u: u \in G(I), m(u)=i\}+i-1$.

Proof. Let $u \in G(I)$ such that $m(u) \geq i$ and $\operatorname{deg} u=d_{i}=m_{i}-(i-1)$. Say, $u=x_{1}^{a_{1}} \cdots x_{n}^{a_{m}}$. Then, since $I$ is stable, we have

$$
v=x_{1}^{a_{1}} \cdots x_{i-1}^{a_{i-1}} x_{i}^{\sum_{t=i}^{n} a_{t}} \in I .
$$

If $v \in G(I)$, then we are done. Suppose there exists $f \in G(I)$ which properly divides $v$; then $\operatorname{deg} f<\operatorname{deg} v=d_{i}$. Hence by the definition of $u$ we must have $m(f)<i$. This implies that $f$ divides $x_{1}^{a_{1}} \cdots x_{i-1}^{a_{i-1}}$. But then $f$ divides $u$, which is a contradiction.

We denote by $b_{i}(I)$ the smallest (with respect to the lexicographic order) element $u \in G(I)$ which has the property that $\operatorname{deg} u=m_{i}-i+1$ and $m(u)=i$. Such an element exists by Lemma 3.3 .

\section{Lemma 3.4.}

$$
m_{i}\left(I, x_{n}\right)=m_{i}(I) \quad \text { for all } i \leq n-1 .
$$

Proof. Since $b_{i}(I) \in\left(I, x_{n}\right)$ it follows from the definition of $b_{i}\left(I, x_{n}\right)$ that $\operatorname{deg} b_{i}(I) \geq$ $\operatorname{deg} b_{i}\left(I, x_{n}\right)$. But it is also clear that $b_{i}\left(I, x_{n}\right) \in I$, so that $\operatorname{deg} b_{i}\left(I, x_{n}\right) \geq \operatorname{deg} b_{i}(I)$. Hence the assertion follows.

Lemma 3.5. There exists a number $t, 0 \leq t \leq n-1$, such that

(a) $m_{i}\left(I: x_{n}\right)=m_{i}(I)$ for $i \leq t$,

(b) $m_{i}\left(I: x_{n}\right) \geq m_{i}(I)-1$ for $t<i \leq n$.

(c) $m_{i}(I)=m_{n}(I)-(n-i)$ for $t<i \leq n$.

Proof. Let $u \in G\left(I: x_{n}\right)$; then $u \in G(I)$ or $u x_{n} \in G(I)$. Indeed, since $u x_{n} \in I$, there exists $g \in G(I)$ such that $g \mid u x_{n}$. If $g=g^{\prime} x_{n}$, then $g^{\prime} \in G\left(I: x_{n}\right)$ and $u=g^{\prime}$; so $u x_{n} \in G(I)$. Otherwise, $g$ divides $u$ in which case $u \in G(I)$ since $G\left(I: x_{n}\right) \cap I \subset G(I)$. It follows that $m_{i}\left(I: x_{n}\right) \geq m_{i}(I)-1$. This implies in particular assertion (b).

We set $d_{i}=\operatorname{deg} b_{i}(I)$ for $i=1, \ldots, n$. We first observe that $d_{i} \leq d_{n}$, and set

$$
t=\min \left\{i: d_{i} \neq d_{n}\right\}-1 \text {. }
$$

Notice that $d_{i}<d_{n}$ for $i \leq t$, and $d_{i}=d_{n}$ for $i>t$. Thus (c) follows trivially from the definition of $t$.

Finally we set $g_{i}=b_{i}\left(I: x_{n}\right)$; then $g_{i} \in G(I)$ or $g_{i} x_{n} \in G(I)$. If $g_{i} \in G(I)$, then $\operatorname{deg} g_{i}=\operatorname{deg} b_{i}$, and $m_{i}\left(I: x_{n}\right)=m_{i}(I)$ for $i \leq t$. If $g_{i} \notin G(I)$, then $g_{i} x_{n} \in G(I)$. It follows that $\operatorname{deg} g_{i}+1 \geq d_{n}>d_{i}$, and hence $\operatorname{deg} g_{i}=d_{i}$. On the other hand, we have $\operatorname{deg} g_{i} \leq d_{i}$ for $i \leq t$, since for these $i, b_{i}(I) \in\left(I: x_{n}\right)$. It follows that $m_{i}\left(I: x_{n}\right)=m_{i}(I)$ for $i \leq t$. This proves (a). 
Lemma 3.6. For all $i$ one has $M_{i}\left(I: x_{n}\right) \leq M_{i}(I)$.

Proof. Let $B(I)=\left\{u_{1}, \ldots, u_{r}, v_{1} x_{n}, \ldots, v_{s} x_{n}\right\}$ with all $u_{i} \in K\left[x_{1}, \ldots, x_{n-1}\right]$ : Then $B\left(I: x_{n}\right) \subset\left\{u_{1}, \ldots, u_{r}, v_{1}, \ldots, v_{s}\right\}$. Therefore we have

$$
\begin{aligned}
M_{i}\left(I: x_{n}\right) & \leq \max \left\{\operatorname{deg} u_{j}, \operatorname{deg} v_{k}: \max \left(u_{j}\right) \geq i, \max \left(v_{k}\right) \geq i\right\}+i-1 \\
& \leq M_{i}(I) .
\end{aligned}
$$

Proof. [Theorem 3.2.] Let $I \subset R$ be stable. Since we may assume that each variable divides one of the generators, it follows from 3.1 that depth $R / I=0$. Hence if $R / I$ is Cohen-Macaulay, as we assume, there exists a pure power of $x_{n}$ belonging to $I$. Let $a>0$ be the smallest integer such that $x_{n}^{a} \in I$. Since $I$ is stable, we conclude that $a \geq \operatorname{deg} u$ for all $u \in G(I)$. Hence we have

$$
M_{i}=a+i-1 \text { for all } i,
$$

and so

$$
e_{i}(R / I) \leq e\left(R /\left(x_{1}, \ldots, x_{n}\right)^{a}\right)=\left(\prod_{i=1}^{n} M_{i}\right) / n !
$$

In order to prove the lower bound we consider the exact sequence

$$
0 \longrightarrow R /\left(I: x_{n}\right) \longrightarrow R / I \longrightarrow R /\left(I, x_{n}\right) \longrightarrow 0 .
$$

As $R / I$ has finite length, the multiplicities of the rings in the above exact sequence is just their length. So, arguing by induction on the length, we have

$$
\begin{aligned}
e(R / I) & =e\left(R /\left(I, x_{n}\right)\right)+e\left(R /\left(I, x_{n}\right)\right) \\
& \geq\left(\prod_{i=1}^{n-1} m_{i}\left(I, x_{n}\right)\right) /(n-1) !+\left(\prod_{i=1}^{n} m_{i}\left(I: x_{n}\right)\right) / n !
\end{aligned}
$$

Therefore it follows from 3.4 and 3.5 that

$$
\begin{aligned}
n ! e(R / I) & \geq n \prod_{i=1}^{n-1} m_{i}+\prod_{i=1}^{t} m_{i} \prod_{i=t+1}^{n}\left(m_{n}-i-2\right) \\
& =n \prod_{i=1}^{n-1} m_{i}+\prod_{i=1}^{t} m_{i} \prod_{i=t+1}^{n-1} m_{i}\left(m_{n}-n+t\right) \\
& =\prod_{i=1}^{n-1} m_{i}\left(m_{n}+t\right) \geq \prod_{i=1}^{n} m_{i} .
\end{aligned}
$$

The upper bound will be proved in the next theorem, more generally, for arbitrary, not necessarily Cohen-Macaulay stable ideals.

For the lower bound of the multiplicity in Theorem 3.2 we need the hypothesis that $R$ is Cohen-Macaulay. Indeed, consider the following example: let $R=$ $K\left[x_{1}, x_{2}, x_{3}, x_{4}\right]$, and $B(I)=\left\{x_{1} x_{2}, x_{2}^{2} x_{4}\right\}$; then

$$
e(R / I)=3, \quad m_{1}=2, \quad m_{2}=3, \quad m_{3}=5, \quad m_{4}=6,
$$

and

$$
3<\frac{2 \cdot 3 \cdot 5 \cdot 6}{24}
$$


Theorem 3.7. For any stable ideal $I$ with $\operatorname{codim} R / I=s$, we have

$$
e(R / I) \leq\left(\prod_{i=1}^{s} M_{i}\right) / s !
$$

Proof. We consider again the exact sequence

$$
0 \longrightarrow R /\left(I: x_{n}\right) \longrightarrow R / I \longrightarrow R /\left(I, x_{n}\right) \longrightarrow 0
$$

By 3.1, we have $\operatorname{dim} R / I=n-\max \{\min (u): u \in I\}$. If $\operatorname{dim} R / I=\operatorname{dim} R /\left(I, x_{n}\right)$, then

$$
n-\max \{\min (u): u \in I\}=(n-1)-\max \{\min (u): u \in \bar{I}\}
$$

where $\bar{I}=\left(I+\left(x_{n}\right)\right) /\left(x_{n}\right) \subset R /\left(x_{n}\right)$. This is only possible if $x_{n}^{a} \in I$ for some integer $a>0$. In this case $R / I$ is Cohen-Macaulay (of $\operatorname{codim} n$ ), and we know the result from 3.2.

If $\operatorname{dim} R / I>\operatorname{dim} R /\left(I, x_{n}\right)$, then $e(R / I)=e\left(R /\left(I: x_{n}\right)\right)$. If $\left(I: x_{n}\right)=I$, then $I$ is an ideal $K\left[x_{1}, \ldots, x_{n-1}\right]$, and we know the result by induction on the number of variables. So we may assume that $I$ is properly contained in $\left(I: x_{n}\right)$, and that $e(R / I)=e\left(R /\left(I: x_{n}\right)\right)$. Let $t=\operatorname{codim} R /\left(I: x_{n}\right)$; then $t \leq s$. Therefore by Noetherian induction and by Lemma 3.6 we have

$$
\begin{aligned}
e(R / I) & =e\left(R /\left(I: x_{n}\right)\right) \\
& \leq\left(\prod_{i=1}^{t} M_{i}\left(I: x_{n}\right)\right) / t ! \leq\left(\prod_{i=1}^{s} M_{i}(I)\right) / s !
\end{aligned}
$$

Recall that the Castelnuovo-Mumford regularity $\operatorname{reg}(I)$ of a graded ideal $I \subset R$ is defined to be the maximum of the numbers $D_{i}(I)=M_{i}(I)-i+1$. Denote by $\operatorname{in}(I)$ the generic initial ideal of $I$ with respect to the reverse lexicographic order. One has $e(R / I)=e(R / \operatorname{in}(I)), \operatorname{reg}(I)=\operatorname{reg}(\operatorname{in}(I))$ and codepth $I=\operatorname{codepth} \operatorname{in}(I)$; see [7] and [6]. Furthermore, in $(I)$ is Borel-fixed, by a theorem of Galligo [10] and Bayer-Stillman [6] (see also [7]).

In case char $K=0$, it follows that $\operatorname{in}(I)$ is stable. On the other hand, if char $K>$ 0 , we consider the polynomial ring $R_{0}$ in $n$ variables over the rational numbers. Let $J_{0} \subset R_{0}$ be the ideal defined by the same monomials as in $(I)$. One has $\beta_{i j}\left(J_{0}\right) \leq$ $\beta_{i j}(\operatorname{in}(I))$ for all $i$ and $j$. This implies that $\operatorname{reg}\left(J_{0}\right) \leq \operatorname{reg}(\operatorname{in}(I))$. Furthermore, we have $e\left(R / J_{0}\right)=e(R / \operatorname{in}(I))$ and $\operatorname{codim}\left(J_{0}\right)=\operatorname{codim}(\operatorname{in}(I))$. Let us denote by $J$, the ideal $\operatorname{in}(I)$ when char $K=0$ and the generic initial ideal of $J_{0}$ with respect to the reverse lexiocographical order, when char $K>0$. Then we get, $e(R / J)=e(R / I)$, $\operatorname{codim}(J)=\operatorname{codim}(I), \operatorname{reg}(J) \leq \operatorname{reg}(I)$ and $J$ is stable. Thus we get

Corollary 3.8. Let $I \subset R$ be a graded ideal of codim $s$. Then

$$
e(R / I) \leq\left(\begin{array}{c}
\operatorname{reg}(I)+s-1 \\
s
\end{array}\right)
$$


Proof. Let $J$ be as above, so that $J$ is stable and $e(R / J)=e(R / I)$. Theorem 3.7, together with the above observations implies that

$$
\begin{aligned}
e(R / I) & =e(R / J) \\
& \leq \prod_{i=1}^{s}\left(\left(D_{i}(J)+i-1\right) / i\right) \\
& \leq \prod_{i=1}^{s}((\operatorname{reg}(I)+i-1) / i) \\
& =\left(\begin{array}{c}
\operatorname{reg}(I)+s-1 \\
s
\end{array}\right) .
\end{aligned}
$$

\section{Squarefree Strongly Stable ideals}

In this section we prove Conjecture 1 for squarefree strongly stable ideals. This class of monomial ideals was first introduced in [2]. Already somewhat before, squarefree lexsegment ideals and their resolution were studied by Evans and Charalambous [8]. Recall that a monomial ideal $I \subset R=K\left[x_{1}, \ldots, x_{n}\right]$ is called squarefree strongly stable if it is generated by squarefree monomials such that for each $u \in G(I)$ we have that $x_{j}\left(u / x_{i}\right) \in I$ for all $j<i$ such that $x_{j}$ does not divide $u$. In particular, the monomial $x_{j}\left(u / x_{i}\right)$ is again squarefree.

Let $I$ be squarefree strongly stable. The graded free $R$-resolution $F$ of $S=R / I$ is very similar to the Eliahou-Kervaire resolution; see [2]. The free module $F_{i}$ has a basis $f(\sigma ; u)$ with $u \in G(I), \sigma \subset\{1, \ldots, n\},|\sigma|=i-1, \max (\sigma)<m(u)$ and $\sigma \cap \operatorname{supp}(u)=\emptyset$ where $\operatorname{supp}(u)$ is the set of indices $i$ such that $x_{i}$ divides $u$. Moreover, one has $\operatorname{deg} f(\sigma ; u)=\operatorname{deg} u+i-1$.

It is immediate from this description that

$$
M_{i}=\max \{\operatorname{deg} u: u \in G(I), \max (u)-\operatorname{deg} u \geq i-1\}+i-1
$$

and

$$
m_{i}=\min \{\operatorname{deg} u: u \in G(I), \max (u)-\operatorname{deg} u \geq i-1\}+i-1 .
$$

Just as for stable ideals we can compute the codepth and codimension of $R / I$ when $I$ is squarefree strongly stable.

Proposition 4.1. Let $I \subset R$ be a squarefree strongly stable ideal. Then

(a) $\operatorname{codim} R / I=\max \{\min (u): u \in I\}=\max \{\min (u): u \in G(I)\}$;

(b) codepth $R / I=\max \{\max (u)-\operatorname{deg}(u): u \in G(I)\}+1$.

Proof. In order to simplify notation we introduce for any monomial ideal $J$ the number

$$
v(J)=\max \{\min (u): u \in G(J)\} .
$$

Since $I$ is squarefree strongly stable it follows that $\left(x_{1}, \ldots, x_{v(I)}\right)$ contains $I$. This implies that $\operatorname{codim}(R / I) \leq v(I)$. Thus, since all minimal prime ideals of a monomial ideal are generated by subsets of $\left\{x_{1}, \ldots, x_{n}\right\}$, it suffices to show that if $P=$ $\left(x_{i_{1}}, \ldots, x_{i_{r}}\right)$ is a prime ideal of $R$ with $I \subset P$, then $r \geq v(I)$.

First suppose that $i_{1} \geq 2$. Write $I=x_{1} I_{1}+I_{2}$ with $I_{1}$ and $I_{2}$ generated by monomials in $K\left[x_{2}, \ldots, x_{n}\right]$. Since $P$ is a prime ideal, and since $x_{1} \notin P$ it follows that $I_{2} \subset P$. Thus, since $I_{2}$ is squarefree strongly stable, induction yields $r \geq v\left(I_{2}\right)$. On the other hand, $v\left(I_{2}\right) \geq v(I)$. This proves the assertion in this case. 
Next suppose that $i_{1}=1$. Let $I^{\prime}=\left(I, x_{1}\right) /\left(x_{1}\right), R^{\prime}=K\left[x_{2}, \ldots, x_{n}\right]$ and $P^{\prime}=\left(P, x_{1}\right) /\left(x_{1}\right)$. Then $P^{\prime}$ is a prime ideal in $R^{\prime}$ with $I^{\prime} \subset P^{\prime}$. By induction on the number of variables we have $r-1 \geq v(I)-1$, and hence $r \geq v(I)$.

(b) follows immediately from the description of the minimal free resolution of $R / I$.

For the proof of the main result of this section we need the following technical

Lemma 4.2. Let $I \subset R$ be a squarefree strongly stable ideal. Then

$$
M_{i}\left(I: x_{n}\right) \leq M_{i}(I) \quad \text { and } \quad \text { codepth } R /\left(I: x_{n}\right) \leq \operatorname{codepth} R / I .
$$

Proof. Let $G(I)=\left\{u_{1}, \ldots, u_{r}, v_{1} x_{n}, \ldots, v_{s} x_{n}\right\}$ with $u_{i}, v_{j} \in K\left[x_{1}, \ldots, x_{n-1}\right]$ for all $i$ and $j$. Then

$$
G\left(I: x_{n}\right) \subset\left\{u_{1}, \ldots, u_{r}, v_{1}, \ldots, v_{s}\right\} .
$$

Next notice that $\max \left(v_{j} x_{n}\right)-\operatorname{deg} v_{j} x_{n} \geq \max \left(v_{j}\right)-\operatorname{deg} v_{j}$ for all $j$, so that

$$
\begin{aligned}
& \text { codepth } R /\left(I: x_{n}\right)-1 \leq \max _{i, j}\left\{\max \left(u_{i}\right)-\operatorname{deg} u_{i}, \max \left(v_{j}\right)-\operatorname{deg} v_{j}\right\} \\
& \quad \leq \max _{i, j}\left\{\max \left(u_{i}\right)-\operatorname{deg} u_{i}, \max \left(v_{j} x_{n}\right)-\operatorname{deg} v_{j} x_{n}\right\} \\
& \quad=\operatorname{codepth} R / I-1 .
\end{aligned}
$$

The inequality for the $M_{i}$ is proved similarly.

Theorem 4.3. Let $I \subset R$ be a squarefree strongly stable ideal with $\operatorname{codim} R / I=s$. Then

$$
e(R / I) \leq\left(\prod_{i=1}^{s} M_{i}\right) / s !
$$

Proof. Let us first suppose that $R / I$ is Cohen-Macaulay. Then

$$
\max \{\max (u)-\operatorname{deg}(u): u \in G(I)\}+1=\max \{\min (u): u \in G(I)\} .
$$

For any monomial $u$ one has $\min (u) \leq \max (u)-\operatorname{deg}(u)+1$. Thus if we choose $v \in G(I) \operatorname{such}$ that $\min (v)$ is maximal, then we must have that $\min (v)=\max (v)-$ $\operatorname{deg}(v)+1$. This implies that $v=x_{m-d+1} x_{m-d+2} \cdots x_{m}$ if $m=\max (v)$ and $d=$ $\operatorname{deg} v$.

We may assume that $x_{n}$ divides one of the elements of $u \in G(I)$. We then claim that $m=n$. Indeed, let $u \in G(I)$ be divisible by $x_{n}$, and let $t=\operatorname{deg} u$.

Assume that $t>d$; since $n-t+1 \leq m-d+1$ (by the choice of $v$ ) it follows that $n-m \leq t-d$. Therefore $u$ has at most $t-d$ factors $x_{i}$ with $i>m$, and hence at least $t-(t-d)=d$ factors $x_{i}$ with $i \leq m$. Since $I$ is stable, and since $v \in I$ it follows that any squarefree monomial $w$ of degree $d$ with $\max (w) \leq m$ belongs to $I$. This implies that $u \notin G(I)$. Thus we must have that $t \leq d$; but then $n-d \leq n-t \leq m-d$, and this implies $m=n$.

Now we are in the situation that $v=x_{n-d+1} \cdots x_{n}$ is an element of $G(I)$. This implies in particular that $I$ contains all squarefree monomials of degree $d$. We now consider the indicator algebra $A=K\left[x_{1}, \ldots, x_{n}\right] /\left(I, x_{1}^{2}, \ldots, x_{n}^{2}\right)$. Obviously one has $\operatorname{dim}_{K} A_{i}=f_{i-1}$ where $f=f_{-1}, f_{0}, f_{1}, f_{2}, \ldots$ is the $f$-vector associated with the simplicial complex $\Delta$ of $I$; see [2].

Notice that $e(R / I)$ is the number of maximal faces of $\Delta$. Thus if $\operatorname{dim} \Delta=\delta$, then $A_{\delta+2}=0$ and $e(R / I)=\operatorname{dim} A_{\delta+1}$. We have just seen that $A_{d}=0$. On the 
other hand $A_{d-1} \neq 0$, since otherwise we would have that $x_{n-d+2} \ldots x_{n} \in I$ which contradicts the fact that $v \in G(I)$. In conclusion we see that

$$
e(R / I)=\operatorname{dim} A_{d-1} \leq\left(\begin{array}{c}
n \\
d-1
\end{array}\right) .
$$

As $M_{i}=d+i-1$ for $i=1, \ldots, n-d+1$ we get

$$
\frac{1}{(n-d+1) !} \prod_{i=1, \ldots, n-d+1} M_{i}=\left(\begin{array}{c}
n \\
d-1
\end{array}\right) \geq e(R / I)
$$

as desired.

Now we treat the general case and proceed as for the stable ideals: consider the exact sequence

$$
0 \longrightarrow R /\left(I: x_{n}\right) \longrightarrow R / I \longrightarrow R /\left(I, x_{n}\right) \longrightarrow 0 .
$$

By 4.1 we have $\operatorname{dim} R / I=n-\max \{\min (u): u \in I\}$. If $\operatorname{dim} R / I=\operatorname{dim} R /\left(I, x_{n}\right)$, then

$$
n-\max \{\min (u): u \in I\}=(n-1)-\max \{\min (u): u \in \bar{I}\},
$$

where $\bar{I}=\left(I+\left(x_{n}\right)\right) /\left(x_{n}\right) \subset R /\left(x_{n}\right)$. This is only possible if $I$ contains an element of the form $x_{i} x_{i+1} \cdots x_{n}$. In this situation however we may proceed in the proof of the Cohen-Macaulay case.

Thus we may assume that $\operatorname{dim} R / I>\operatorname{dim} R /\left(I, x_{n}\right)$. Then, using 4.2 , the proof is word by word the same as in the case of stable ideals; cf. the proof of 3.7 .

Now we turn to the proof of the lower inequality, and first observe

Remark 4.4. Let $I \subset R$ be a squarefree strongly stable ideal. Then

$$
G\left(I: x_{n}\right) \subset\{u: u \in G(I), \max u \neq n\} \cup\left\{u / x_{n}: u \in G(I), \max u=n\right\},
$$

and

$$
G(\bar{I})=\{u: u \in G(I), \max u \neq n\} .
$$

Lemma 4.5. Let $R / I$ be Cohen-Macaulay, and let $s=\operatorname{codepth} R / I$. Then there exists an integer $t, 0 \leq t<n$, such that

$$
m_{i}(I)<n-s+i, \quad \text { for } \quad i<t \quad \text { and } \quad m_{i}(I)=n-s+i, \quad \text { for } \quad i \geq t .
$$

Proof. Since $R / I$ is Cohen-Macaulay, there exist $u=x_{i} x_{i+1} \cdots x_{n}$ in $G(I)$ for some $i$. By Proposition 4.1, we have $i=s$, so that $v=x_{s} \cdots x_{n} \in I$. Thus,

$$
m_{i}(I) \leq n-s+1+i-1=n-s+i
$$

for all $i$. Let $t$ be such that $m_{i}(I)<n-s+i$ for all $i<t$ and $m_{t}(I)=n-s+t$. For $i>t$, let $u$ be of minimal degree in $G(I)$ with $\max u-\operatorname{deg} u \geq i-1$. Then $\operatorname{deg}(u)+t-1 \geq m_{t}(I)=n-s+t$. This implies that $\operatorname{deg}(u) \geq n-s+1$ and $m_{i}(I) \geq n-s+1+i-1=n-s+i$. Thus, $m_{i}(I)=n-s+i$ for all $i \geq t$.

Lemma 4.6. Let $s$ and $t$ be the numbers introduced in 4.5. Then

$$
m_{i}(\bar{I})=m_{i}(I), \quad \text { for } \quad i \leq s-1,
$$




$$
\begin{aligned}
& m_{i}\left(I: x_{n}\right) \geq m_{i}(I)-1, \quad \text { for } \quad i<t \quad \text { and } \\
& m_{i}\left(I: x_{n}\right)=n-s+i-1, \quad \text { for } i \geq t .
\end{aligned}
$$

Proof. Since $G(I) \subset G(\bar{I}), m_{i}(\bar{I}) \geq m_{i}(I)$ for all $i \leq s-1$. But, if $u$ in $G(I)$ is divisible by $x_{n}$, then either $u^{\prime}=\left(u / x_{n}\right) x_{i} \in G(I)$ for some $i$, or $\operatorname{deg}(u)+i-1>$ $m_{i}(I)$. This implies that $m_{i}(\bar{I}) \leq m_{i}(I)$, and hence $m_{i}(\bar{I})=m_{i}(I)$.

Now, suppose that $m_{i}(I)=n-s+i$ so that $v=x_{s} \cdots x_{n}$ is an element of minimal degreee in $\{u: \max (u)-\operatorname{deg}(u) \geq i-1, u \in G(I)\}$. Then $v^{\prime}=x_{s} \cdots x_{n-1} \in\left(I: x_{n}\right)$ and $\max v^{\prime}-\operatorname{deg} v^{\prime} \geq i-1$. The minimality of $v$ forces $v^{\prime}$ to be in $G\left(I: x_{n}\right)$. So,

$$
m_{i}\left(I: x_{n}\right)=m_{i}(I)-1=n-s+i-1, \quad i \geq t .
$$

Since, $v \in G(I)$ and $I$ is squarefree strongly stable, no monomial of smaller degree in $G(I)$ is a multiple of $x_{n}$. So, for $i<t$, an element of minimal degree, say $d_{i}$, in $\{u: \max (u)-\operatorname{deg}(u) \geq i-1, u \in G(I)\}$ is not a multiple of $x_{n}$. Hence $d_{i}$ is the minimal degree in $\left\{u: \max (u)-\operatorname{deg}(u) \geq i-1, u \in G\left(I: x_{n}\right)\right\}$ as well, and so

$$
m_{i}\left(I: x_{n}\right)=m_{i}(I), \quad i<t .
$$

Theorem 4.7. Let $I \subset R$ be a squarefree strongly stable such that $R / I$ is CohenMacaulay. Then

$$
e(R / I) \geq\left(\prod_{i=1}^{n} m_{i}\right) / s !
$$

Proof. Consider the exact sequence

$$
0 \longrightarrow R /\left(I: x_{n}\right) \longrightarrow R / I \longrightarrow R /\left(I, x_{n}\right) \longrightarrow 0 .
$$

By the Proposition 4.1 and Remark 4.4, these rings all have the same dimension unless $R / I=K$, in which case we already know the result. Since the multiplicity is additive,

$$
e(R / I)=e\left(R / I: x_{n}\right)+e\left(R /\left(I, x_{n}\right)\right) .
$$

Since $R / I$ is Cohen-Macaulay of codimension $s, v=x_{s} \cdots x_{n}$ is in $G(I)$. If $R /\left(I, x_{n}\right)$ is not Cohen-Macaulay, then codepth of $R /\left(I, x_{n}\right)$ is less than $s-1$ and $v x_{s-1} / x_{n}=$ $x_{s-1} \cdots x_{n-1}$ is not in $G(I)$. So, for all $i \leq s, m_{i}(I)<n-s+i$. In other words, the integer $t$ in Lemma 4.5 is actually greater than $s$. Hence by Lemma 4.6, $m_{i}(I)=m_{i}\left(\left(I: x_{n}\right)\right)$ for all $i \leq s$. Then by induction,

$$
e(R / I) \geq e\left(R /\left(I: x_{n}\right)\right) \geq \prod_{i=1}^{s} m_{i}\left(I: x_{n}\right)=\prod_{i=1}^{s} m_{i}(I),
$$

as desired.

On the other hand, if $R /\left(I, x_{n}\right)$ is Cohen-Macaulay, then by induction, we get

$$
e(R / I) \geq \frac{\prod_{i=1}^{s} m_{i}\left(I: x_{n}\right)}{s !}+\frac{\prod_{i=1}^{s-1} m_{i}(\bar{I})}{(s-1) !}
$$


and hence

$$
\begin{aligned}
s ! e(R / I) & \geq \prod_{i=1}^{s} m_{i}\left(I: x_{n}\right)+s \prod_{i=1}^{s-1} m_{i}(\bar{I}) \\
& =\prod_{i=1}^{t-1} m_{i}(I) \prod_{i=t}^{s}(n-s+i-1)+s \prod_{i=1}^{t-1} m_{i}(I) \prod_{i=t}^{s-1}(n-s+i) \\
& =\prod_{i=1}^{t-1} m_{i}(I) \prod_{i=t}^{s-1}(n-s+i)(n-s+t+s) \\
& \geq \prod_{i=1}^{s} m_{i}(I) .
\end{aligned}
$$

\section{REFERENCES}

1. A. Aramova and J. Herzog. Koszul cycles and Eliahou-Kervaire type resolutions. J. Algebra 181 (1996), 347-370. MR 97c:13009

2. A. Aramova, J. Herzog and T. Hibi. Squarefree lexsegment ideals. Preprint 1995.

3. D. Buchsbaum and D. Eisenbud. Algebra structures for finite free resolutions, and some structure theorems for ideals of codim 3. Amer. J. Math. 99 (1977), 447-485. MR 56:11983

4. W. Bruns and J. Herzog. Cohen-Macaulay Rings. Cambridge University Press, 1993. MR 95h: 13020

5. W. Bruns and J. Herzog. On multigraded resolutions. Math. Proc. Cambridge Phil. Soc. 118 (1995), 245-257. MR 96g:13013

6. D. Bayer and M. Stillman. A criterion for detecting m-regularity. Invent. Math. 87 (1987), 1-11. MR 87k:13019

7. D. Eisenbud. Commutative Algebra with a view to algebraic geometry. Springer-Verlag, 1995.

8. G. Evans and H. Charalambous. Resolutions obtained by iterated mapping cones. J. Algebra 176 (1995), 750-754. MR 96h:13034

9. S. Eliahou and M. Kervaire. Minimal resolutions of some monomial ideals. J. Algebra 129 (1990), 11-25. MR 91b:13019

10. A. Galligo. A propos du théorème de préparation de Weierstrass. In: Fonctions des Plusieurs Variables Complexes. Lecture Notes in Math., vol. 409, Springer, 1974, pp. 543-579. MR 53:5924

11. J. Herzog, A. Simis and W. V. Vasconcelos. Approximation complexes of blowing-up rings, II. J. Algebra 82(1) (1983), 53-83. MR 85b:13015

12. J. Herzog, N. V. Trung and G. Valla. On hyperplane sections of reduced irreducible varieties of low codimension. J. Math. Kyoto Univ. 34(1) (1994), 47-72. MR 95d:14048

13. C. Huneke and M. Miller. A note on the multiplicity of Cohen-Macaulay algebras with pure resolutions. Canad. J. Math. 37 (1985), 1149-1162. MR 87d:13024

14. C. Peskine and L. Szpiro. Syzygies and multiplicities. C. R. Acad. Sci. Paris. Sér. A 278 (1974), 1421-1424. MR 50:2152

15. N. Terai, Generalization of Eagon-Reiner theorem and $h$-vectors of graded rings. Preprint 1997.

FB 6 Mathematik und Informatik, Universität-GHS-Essen, Postfach 103764, Essen 45117, Germany

E-mail address: mat300@uni-essen.de

Department of Mathematics, University of Missouri, Columbia, Missouri 65211

E-mail address: mathhs@mizzoui.missouri.edu 